\title{
Regulation of Ov2 by virus encoded microRNAs
}

\author{
Katie Nightingale $^{1,2} \cdot$ Inga Dry $^{1} \cdot$ John Hopkins $^{1} \cdot$ Robert Dalziel $^{1}$
}

Received: 28 February 2019 / Accepted: 7 March 2019 / Published online: 19 March 2019

(C) The Author(s) 2019

\begin{abstract}
Herpesviruses encode miRNAs that target both virus and host genes; however their role in herpesvirus biology is still poorly understood. We previously identified thirty five miRNAs encoded by OvHV-2; the causative agent of malignant catarrhal fever (MCF) and are investigating the role of these miRNAs in regulating expression of OvHV-2 genes that play important roles in virus biology. Analysis, using RNAHybrid predicted that two OvHV-2 encoded miRNAs, ovhv2-miR-17-10 and ovhv2-miR-611, target transcripts coding for the OvHV-2 bZIP protein Ov2. In other herpesvirus bZIP proteins are known to play important roles in lytic virus replication. Here we show by Flow cytometry and western blotting that ovhv2-miR-17-10 and ovhv2-miR-611 , reduce the expression of Ov2 protein. The predicted target sites for both miRNAs within the Ov2 gene were disrupted whilst retaining the Ov2 coding sequence. Mutation of the ovhv2-miR-61-1 target sequence restored Ov2 protein expression levels to control levels confirming the identity of its target site. However, it was not possible to determine the binding site of ovhv2-miR17-10 possibly due to potential G:U pairing introduced during the mutation process. The targeting of Ov2 by two virus-encoded miRNAs suggests an important regulatory role for Ov2 in OvHV-2 replication or reactivation.
\end{abstract}

Keywords Ovine herpesvirus-2 · Virus encoded miRNAs, Ov2

\section{Introduction}

Malignant catarrhal fever (MCF) is a usually fatal disease of cattle, deer, bison and other ruminants caused by viruses in the genus Macavirus of the subfamily Gammaherpesvirinae (McGeoch et al. 2006). MCF is characterised by sudden onset of fever followed by lymphadenopathy, leucocytosis, severe congestion and necrosis and erosion of the oral, conjunctival and nasal muscosæ (Russell et al. 2009). The major causative agents of MCF are ovine herpesvirus 2 (OvHV-2) and alcelaphine herpesvirus 1 (AlHV-1), which cause the sheep associated (SA-MCF) and the wildebeest associated (WAMCF) forms of the disease, respectively. In the case of both the SA and WA- forms of MCF, reactivation of the virus from latency results in the production of infectious virions that can

Robert Dalziel

bob.dalziel@roslin.ed.ac.uk

1 The Roslin Institute \& R(D)SVS, University of Edinburgh, Edinburgh, Midlothian EH25 9RG, UK

2 Present address: Cambridge Institute of Medical Research, Hills Road, Cambridge CB2 0XY, UK be transmitted to susceptible species, such as cattle, bison, or deer found in close proximity to asymptomatically shedding carrier animals.

Reactivation of gammaherpesviruses from their latent state requires the expression of viral transactivators, such as the Replication and Transcriptional Activator protein (RTA), that promote virus replication by triggering expression of a cascade of proteins that are required for viral genomic replication and particle formation (Damania et al. 2004). The Ov2 gene of OvHv-2 encodes a protein with a basic leucine zipper (bZIP) domain. Herpesvirus proteins with bZIP domains include BZLF1 of Epstein Barr Virus (EBV) (Murata 2014), and the K8 protein of Kaposi's sarcoma-associated herpesvirus (KSHV) (Lefort and Flamand 2009). BZLF and K8 play important roles in the lifecycles of EBV and KSHV by modulating the activity of RTA (Liao et al. 2003; Murata 2014).

MicroRNAs (miRNAs) are short, non-coding RNAs that regulate gene expression at a post-transcriptional level. miRNA-mediated regulation of expression is by binding of the seed sequence (usually nucleotides 2 to 8 ) to complementary sequences in the target mRNA and directing these targets for degradation or translational silencing, depending on the degree of complementarity observed (Bartel 2009). 
MicroRNAs have been identified in the genomes of many virus families, though, it is the Herpesviridae, with over 300 viral microRNAs identified so far amongst its members, that seem to have evolved to make the greatest use of miRNAs as a mechanism to effectively regulate cellular and virus gene expression (Grundhoff and Sullivan 2011). Independent research into the targets of the miRNAs encoded by herpesviruses have identified in the genomes of viruses classified within all herpesvirus sub-families, the presence of miRNAs which target the viral transactivators (Bellare and Ganem 2009; Grey et al. 2007; Umbach et al. 2008). This is indicative of a familial level mechanism regulating the balance between herpesvirus lytic and latent replication cycles.

Previously our group has shown that OvHV-2 encodes for at least thirty-five miRNAs, and that the targets of these miRNAs include transcripts encoding the OvHV-2 RTA (encoded by the ORF50 transcript) (reactivation) and ORF73 (maintenance of latency) (Nightingale et al. 2014; Riaz et al. 2014). Further analysis, using RNAHybrid (Rehmsmeier et al. 2004) predicted that two further OvHV-2 encoded miRNAs, ovhv2-miR-17-10 and ovhv2-miR-61-1, would target transcripts coding for the OvHV-2 protein Ov2.

In this paper, we show that two of the thirty-five identified OvHV-2-encoded miRNAs, ovhv2-miR-17-10 and ovhv2miR-61-1, target the virus-encoded protein Ov2. Furthermore, the fact that two virus-encoded miRNAs target Ov2 is suggestive of an important regulatory role for Ov2 in the process of OvHV-2 reactivation.

\section{Methods}

\section{Cell culture}

BJ1035 cells, an immortalized bovine $\mathrm{T}$ cell line from an animal naturally-infected with OvHV-2 [3], was grown in suspension culture in Iscove's Modified Dulbecco's Medium (Invitrogen, UK) supplemented with $10 \%(v / v)$ foetal calf serum (FCS, Sera Laboratories International, UK), $1 \%$ (v/v) penicillin-streptomycin (Invitrogen), $20 \mathrm{U} / \mathrm{ml}$ interleukin 2 (Novartis Pharmaceuticals UK) and incubated at $37{ }^{\circ} \mathrm{C}, 5 \%$ $\mathrm{CO}_{2}$. Human embryonic kidney cells (HEK-293 T) were cultured in Dulbecco's Modified Eagle Medium (Invitrogen) supplemented with $10 \%(\mathrm{v} / \mathrm{v})$ FCS and $1 \%(\mathrm{v} / \mathrm{v})$ penicillinstreptomycin-glutamine (Invitrogen) and incubated at $37{ }^{\circ} \mathrm{C}$, $5 \% \mathrm{CO}_{2}$.

\section{Target identification}

The nucleotide sequence of Ov2 was derived from the OvHv2 genome (Genbank accession: AY839756.1). Sequences and position of the OvHV-2-encoded miRNA in the OvHV-2 genome have been described previously (Nightingale et al.
2014). RNAHybrid (Rehmsmeier et al. 2004) was used to predict OvHV-2-encoded miRNA target sites within the coding region of Ov2. No G:U pairing in the seed sequence was allowed, and a helix constraint of nucleotides 2 to 8 was used.

\section{Primer design and cloning}

The OvHV-2 OV2 gene sequence amplified by PCR from DNA extracted from OvHV-2 positive BJ1035 cells (Schock et al. 1998) using the DNA Blood and Tissue kit (Qiagen, UK). The reaction contained 1 unit HotStarTaq Plus DNA polymerase (Qiagen), $50 \mathrm{ng}$ BJ1035 DNA, $200 \mu \mathrm{M}$ dNTPS and 8 pmols of each primer in a final reaction volume of $20 \mu \mathrm{l}$.

The cycling conditions used for amplification were an initial denaturation step of $5 \mathrm{~min}$ at $95^{\circ} \mathrm{C}$, followed by 30 cycles of $95^{\circ} \mathrm{C}$ for $30 \mathrm{~s}, 58^{\circ} \mathrm{C}$ for $1 \mathrm{~min}$ and $72^{\circ} \mathrm{C}$ for $1 \mathrm{~min}$, with a final extension of $72^{\circ} \mathrm{C}$ for $7 \mathrm{~min}$.

Untagged Ov2 was cloned into pcDNA3.1(+) using the restriction enzymes BamH1 and EcoR1. Ov2 was cloned in frame with EGFP, in the vector pEGFPN1, using the restriction enzymes $B g l I I$ and EcoRl. Sequencing by GATC (Cologne, Germany) confirmed the absence of any additional mutations in the Ov2 sequence.

\section{Site directed mutagenesis}

Site-directed mutagenesis PCR was carried out using the QuikChange Lightning Site-Directed Mutagenesis Kit (Agilent Technologies, UK) according to the manufacturer's protocol. Mutagenesis primers (Table 1) were designed using the QuikChange Primer Design Program (www.agilent.com/ genomics/qcpd). All mutations were confirmed by sequencing by GATC.

\section{Flow cytometry}

Ten ng plasmid DNA and $100 \mathrm{nM}$ miRNA mimic (Nightingale et al. 2014) or $100 \mathrm{nM}$ AllStars Negative Control siRNA (both Qiagen) were transfected into HEK293 T seeded at $3 \times 10^{4}$ cells per well of a 96 well dish, using Lipofectamine 2000 (Invitrogen). Cells were detached using trypsin $48 \mathrm{~h}$ after transfection, washed and resuspended in $1 \%$ $(w / v)$ PBS/BSA. Samples were analysed using an LSRFortessa High Throughput Sampler (BD Biosciences). Forward scatter and side scatter were used to gate live cells. A mock transfected cell population was used as a negative control. Data were analysed using FlowJo v10.0.6. Median fluorescence intensity (MFI) was used as a measure of expression. 7500 GFP-positive cells were counted with $n=14$ for each condition. 


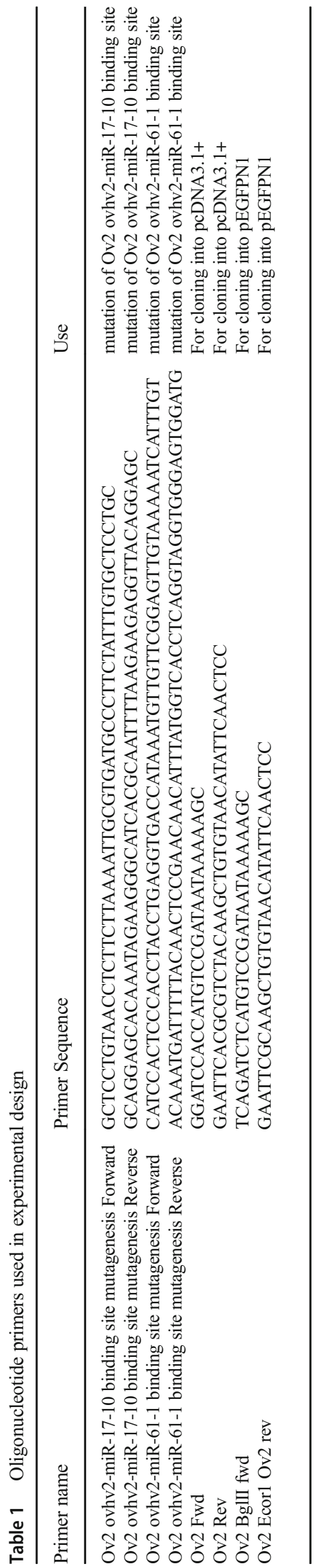

\section{Western blotting}

One hundred ng plasmid DNA and $100 \mathrm{nM}$ miRNA mimic (Nightingale et al. 2014) or $100 \mathrm{nM}$ AllStars Negative Control siRNA (both Qiagen) were transfected using Lipofectamine 2000 , into $2 \times 10^{5}$ HEK-293 T cells per well of a 12 well dish. Samples were harvested in Laemmli Buffer (Bio-Rad, UK) and boiled for $5 \mathrm{~min}$ at $95{ }^{\circ} \mathrm{C}$ before separation by SDS-PAGE using 4-20\% Mini-PROTEAN® TGX ${ }^{\mathrm{TM}}$ Gels (Bio-Rad). Membranes were probed with either rabbit anti-Ov2 antibody (synthesised using peptides RRSTNRRASRNFKKRLQEH (amino acids 26-45, accession \# AY839756.1) and EKKKEEIRRLQYWLSTHNC, (amino acids 67-85 accession \# AY839756.1) peptides by Dundee Cell Products, UK) or a mouse anti-beta Actin (mAbcam 8226) and appropriate fluorescent secondary antibodies (LI-COR). Primary (1:1000) and Secondary antibodies $(1: 10000)$ were diluted in PBS-T (PBS $+0.1 \%$ Tween $20 \mathrm{v} / \mathrm{v}$ ). Protein bands were visualised using an Odyssey CLx (LI-COR) and quantified using Image Studio Lite version 3.1 (LI-COR). For each sample, the levels of Ov2 were quantified and normalised to the level of actin. Test ovhv2-miRNA samples were then compared to the negative control siRNA. A total of three independent western blots were performed with duplicates for each condition.

\section{Statistical analysis}

Statistical analysis was performed using Minitab 17 software. Differences between groups were analysed using a general linear model followed by Tukey's post-hoc test. $P$-values represent results from the post-hoc test.

\section{Illustrations}

Illustrations were generated using GraphPad Prism.

\section{Results}

\section{Control of Ov2 expression by OvHV-2 encoded miRNAs}

The capacity of two OvHV-2-encoded miRNAs (ovhv2-miR$17-10$ (minimum free energy, $\mathrm{mfe}=-23.8 \mathrm{kcal} / \mathrm{mol}$ ) and ovhv2-miR-61-1 $(\mathrm{mfe}=-30.7 \mathrm{kcal} / \mathrm{mol})$, Fig. 1$)$, to affect Ov2 expression was investigated .

HEK-293 T cells were transfected with Ov2-EGFPN1 and either ovhv2-miR-17-10 mimic, ovhv2-miR-61-1 mimic, a mixture of both or a negative control siRNA. Forty-eight hours post transfection GFP expression was analysed by flow 


\section{a ovhv2-miR-61-1 binding site:}

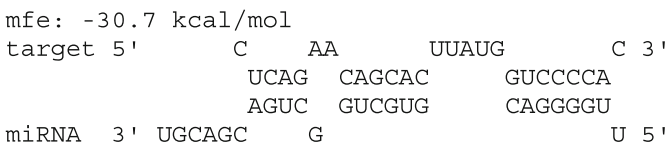

mutated ovhv2-miR-61-1 binding site:

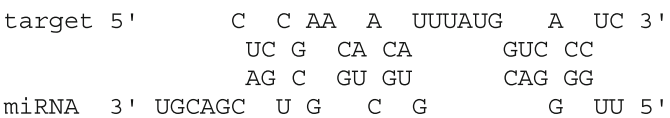

b ovhv2-miR-17-10 binding site:

mfe: $-23.8 \mathrm{kcal} / \mathrm{mol}$

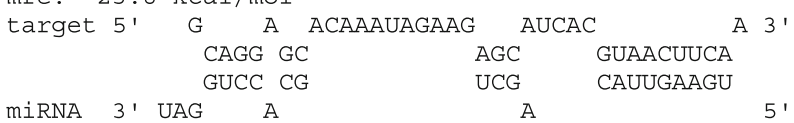

mutated ovhv2-miR-17-10 binding site:

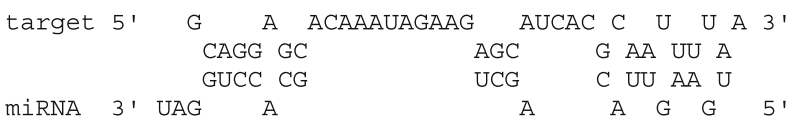

Fig. 1 RNAHybrid analysis showing predicted binding of ovhv2miR- 61-1 and ovhv2-miR-17-10 to Ov2 target sequence. RNAHybrid analysis showing predicted binding, with minimum free energys (mfe) of ovhv2-miR- 61-1(a) and ovhv2-miR-17-10 (b) to the Ov2 target sequence. The RNAHybrid analysis showing predicted binding ovhv2-miR-61-1 (a) and ovhv2-miR-17-10 (b) to a mutated Ov2 target sequence is shown underneath. Constraints on miRNA binding included perfect complementarity between nucleotides 2 and 8 and no G:U pairing within this region

cytometry as described above. Overall, the percentage of GFP positive cells was found to remain consistent between replicates and conditions (data not shown). To control for off-target effects, the ability of the OvHV-2 miRNA mimics to reduce expression of GFP alone was also examined. No significant changes in the MFI were observed when ovhv2-miR-17-10 or

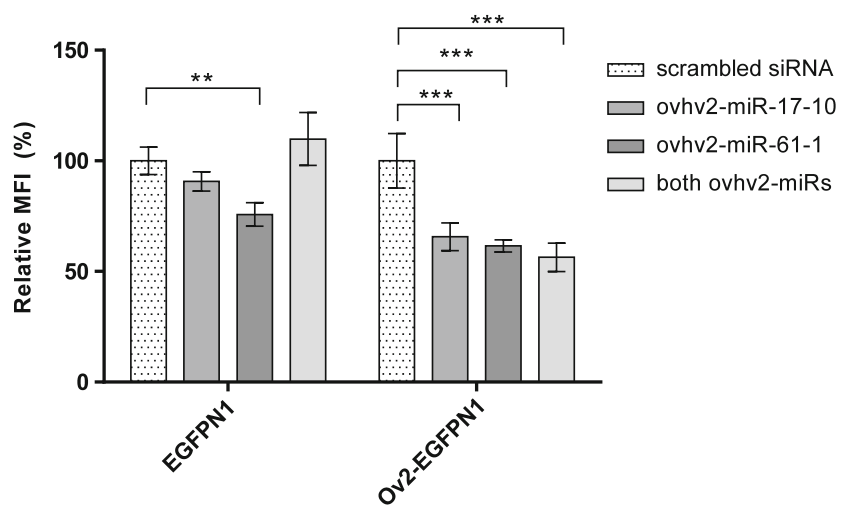

Fig. 2 Confirmation of Ov2 targeting by miRNAs using Flow cytometry. 293 T cells were co-transfected with either an EGFPN1 or an Ov2-EGFPN1 construct and ovhv2-miR-17-10, ovhv2-miR-61-1, a combination of both ovhv2-miRs or a scrambled siRNA. The MFI for each sample was determined $48 \mathrm{~h}$ later by flow cytometry and test ovhv2-miRNAs were compared to a scrambled siRNA control. $* *=p \leq 0.01$, $* * *=p \leq 0.0001$. Error bars show \pm SEM. MFI $=$ Mean Fluorescence Intensity a combination of both ovhv2-miRs were co-transfected with an EGFPN1 construct compared to a negative control siRNA (Fig. 2). A decrease of $25 \%(p \leq 0.01)$ in the MFI was observed when ovhv2-miR-61-1 was co-transfected with an EGFPN1 construct compared to the negative control siRNA (Fig. 2). Significant decreases ( $p \leq 0.0001$ for all) of $35 \%$, $39 \%$ and $44 \%$ in the MFI were observed when ovhv2-miR17-10, ovhv2-miR-61-1 or a combination of both ovhv2-miRs respectively were co-transfected with an Ov2- EGFPN1 construct compared to a negative control siRNA (Fig. 2).

As there were potential off target effects of the ovhv2-miR61-1 on EGFP we investigated the effects of the OvHv-2 miRNA mimics to regulate expression of an untagged version of Ov2, by western blot analysis using an Ov2 specific antibody. Using this approach, the ability of either ovhv2-miR-1710 or the ovhv2-miR-61-1 to reduce expression of Ov2 transcripts that had either a disrupted ovhv2-miR-17-10 or ovhv2miR-61-1 binding site was also examined. In each case the

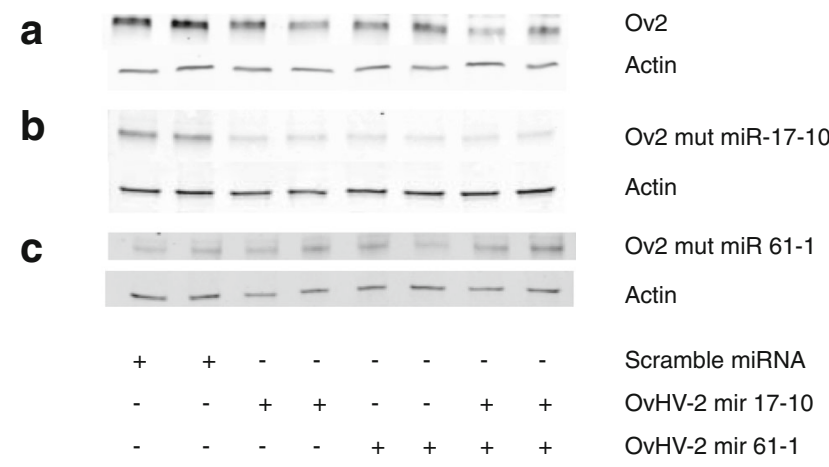

d

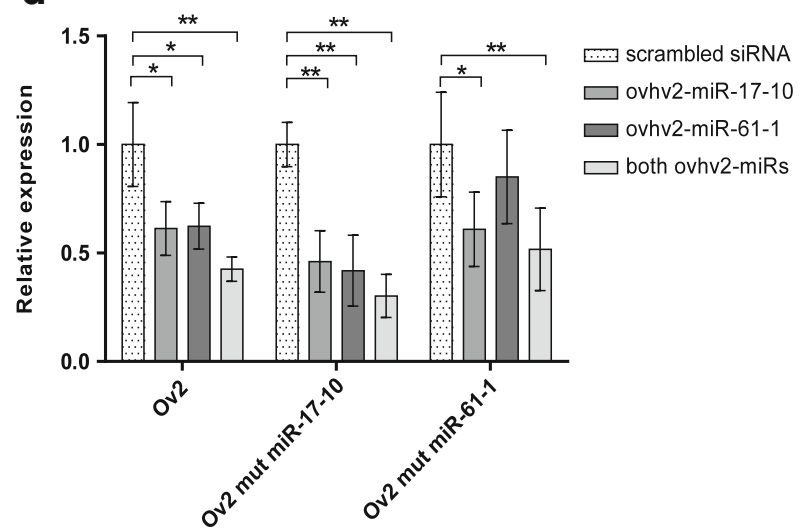

Fig. 3 Analysis of miRNAs targeting Ov2 by western blotting. HEK293 T were transfected with the expression construct Ov2-pcDNA3.1+ and the appropriate miRNA mimic or a scramble siRNA. Representative western blots of protein extracts from cells transfected with (a) Ov2pcDNA3.1+, (b) mutated Ov2-mut -mIR-17-10 or (c) Ov2-mut-miR61-1 with ovhv2-mIR-17-10, ovhv2-miR-61-1 or a combination of both are shown. (d) Ov2 was detected using an anti-Ov2 antisera and quantified using the LI-COR system. Expression of Ov2 was normalised to expression of actin for each sample. Levels of expression in the presence of test ovhv2-miRs were compared to a scrambled siRNA. $*=p \leq 0.05$, $* *=p \leq 0.01, * * *=p \leq 0.0001$ 
mutagenesis was designed to mutate the binding site, without impacting on the coding sequence of Ov2 (Fig. 1).

When either ovhv2-miR-17-10 or ovhv2-miR-61-1 were co-transfected with an Ov2- pcDNA3.1(+) construct significant reductions of approximately $40 \%$ ( $p \leq 0.05$ for both ovhv2-miRNAs) were observed compared to the negative control siRNA. A reduction of approximately $60 \%$ was observed compared to the negative control siRNA when both ovhv2-miR-17-10 and ovhv2-miR-61-1 were co-transfected with the Ov2- pcDNA3.1(+) construct $(p \leq 0.01)$. Restoration of expression of Ov2 was observed when ovhv2-miR-61-1 was co-transfected with Ov2-mut-miR-61$1(p \leq 0.58)$, though this construct remained sensitive to the presence of ovhv2-miR-17-10 ( $p \leq 0.05$; Fig. 3). However, restoration of Ov2 expression was not observed when Ov2mut-miR-17-10 was expressed in the presence of either ovhv2-miR-17-10 or ovhv2-miR-61-1 ( $p \leq 0.01$; Fig. 3).

\section{Discussion}

Evidence is accumulating from a number of herpesvirus, including HCMV and KSHV, that virally-encoded miRNAs may function like a rheostat, in fine-tuning the mechanism of reactivation by targeting viral transactivators (Grey et al. 2007; Bellare and Ganem 2009; Riaz et al. 2014). A similar role for miRNAs, in increasing stringency to biological pathways, has also been postulated for cellular miRNAs (Ebert and Sharp 2012). In this study, ovhv2-miR-17-10 and ovhv2-miR-61-1 have been shown to target Ov2. Mutation of the target seed sequence of ovhv2miR-61-1 restored Ov2 expression levels to that of the control confirming that the target site of ovhv2-miR-61-1, within the coding sequence of the Ov2 mRNA, had been successfully identified. However, it was not possible to determine the functional binding site of ovhv2-miR-17-10. It is known that functional miRNAs can have G:U pairing within the seed sequence (Didiano and Hobert 2006; Doench and Sharp 2004). To disrupt ovhv2-miR-17-10 binding, three amino acids (an arginine, an asparagine and a phenylalanine) were mutated. There are only two codons available for asparagine (AAC and AAT) and phenylalanine (TTC and TTT) and due to constraints of the genetic code mutation of these two amino acids results in changes in the base pairing of ovhv2-miR-17-10 with Ov2 from G:C to G:U. G:U pairing is allowed in seed sequences and can still result in functional knockdown of the target. Furthermore, RNAhybrid (Rehmsmeier et al. 2004) analysis using ovhv2-miR-17-10, allowing for $\mathrm{G}: \mathrm{U}$ pairing and perfect complementarity between the Ov2 coding sequence and the miRNA seed sequence, identified two further possible and non-overlapping sites for ovhv2miR-17-10 binding, in addition to the site tested. It is therefore possible that the reason Ov2 expression could not be restored, by mutation of the ovhv2-miR-17-10 binding site, is due both to the presence of functionally redundant ovhv2-miR-17-10 binding sites within the Ov2 mRNA or that ovhv2-miR-17-10 is still able to interact with the mutated Ov2 binding site via non-canonical G:U pairing.

Amongst cellular mRNAs, it has been shown that those that encode regulatory proteins, such as transcription factors, have a short half-life (Sharova et al. 2009; Yang et al. 2003). That Ov2 has a short half-life most likely explains the difficulties faced in detecting Ov2 transcript, despite being able to routinely detect protein and DNA in transfected cells. As a result it remains unclear whether ovhv2-miR-17-10 and ovhv2-miR-61-1 function to inhibit Ov2 expression via translational repression or transcriptional degradation. Previously, we have reported that ovhv2-miR-17-10 targets the OvHV-2 RTA transcript (Riaz et al. 2014). The observation that Ov2 is targeted by at least two viral-encoded miRNAs indicates that it has an important regulatory role in OvHV-2 biology. Using a reporter assay system, we have been able to demonstrate that Ov2 is capable of modulating RTA (See accompanying paper Dry et al. 2019). We propose that OvHV-2 utilises miRNAs ovhv2-miR-5 (Riaz et al. 2014), ovhv2-miR-17-10 and ovhv2-miR-61-1 to post-transcriptionally maintain latency, by restricting expression of RTA and Ov2, respectively, thereby increasing stringency to the process of reactivation by preventing precocious initiation of the productive lytic cycle.

Acknowledgements We would like G.C.Russell of the Moredun Research Institute for providing us with the BJ1035 cell-line.

Funding This work was funded under the BBSRC programs: BBS/E/D/ 20241864 and BBS/E/D/20002173. KN was supported by a University of Edinburgh Principals Scholarship.

\section{Compliance with ethical standards}

Conflict of interest The authors declare that they have no conflict of interest.

Open Access This article is distributed under the terms of the Creative Commons Attribution 4.0 International License (http:// creativecommons.org/licenses/by/4.0/), which permits unrestricted use, distribution, and reproduction in any medium, provided you give appropriate credit to the original author(s) and the source, provide a link to the Creative Commons license, and indicate if changes were made.

\section{References}

Bartel DP (2009) MicroRNAs: target recognition and regulatory functions. Cell 136:215-233

Bellare P, Ganem D (2009) Regulation of KSHV lytic switch protein expression by a virus-encoded microRNA: an evolutionary adaptation that fine-tunes lytic reactivation. Cell Host Microbe 6:570-575

Damania B, Jeong JH, Bowser BS, DeWire SM, Staudt MR, Dittmer DP (2004) Comparison of the Rta/Orf50 transactivator proteins of gamma-2-herpesviruses. J Virol 78:5491-5499

Didiano D, Hobert O (2006) Perfect seed pairing is not a generally reliable predictor for miRNA-target interactions. Nat Struct Mol Biol 13:849-851 
Doench JG, Sharp PA (2004) Specificity of microRNA target selection in translational repression. Genes Dev 18:504-511

Dry I, Nightingale K, Ferguson J, Hopkins J, Dalziel R (2019) Ov2 is a modulator of OvHV-2 RTA mediated gene expression. Vet Res Commun. https://doi.org/10.1007/s11259-019-09748-w

Ebert MS, Sharp PA (2012) Roles for microRNAs in conferring robustness to biological processes. Cell 149:515-524

Grey F, Meyers H, White EA, Spector DH, Nelson J (2007) A human cytomegalovirus-encoded microRNA regulates expression of multiple viral genes involved in replication. PLoS Pathog 3:e163

Grundhoff A, Sullivan CS (2011) Virus-encoded microRNAs. Virology 411:325-343

Lefort S, Flamand L (2009) Kaposi's sarcoma-associated herpesvirus KbZIP protein is necessary for lytic gene expression, DNA replication, and virion production in primary effusion lymphoma cell lines. J Virol 83:5869-5880

Liao W, Tang Y, Lin SF, Kung HJ, Giam CZ (2003) K-bZIP of Kaposi's sarcoma-associated herpesvirus/human herpesvirus 8 (KSHV/HHV8) binds KSHV/HHV-8 Rta and represses Rta-mediated transactivation. J Virol 77:3809-3815

McGeoch DJ, Rixon FJ, Davison AJ (2006) Topics in herpesvirus genomics and evolution. Virus Res 117:90-104

Murata T (2014) Regulation of Epstein-Barr virus reactivation from latency. Microbiol Immunol 58:307-317

Nightingale K, Levy CS, Hopkins J, Grey F, Esper S, Dalziel RG (2014) Expression of ovine herpesvirus -2 encoded microRNAs in an immortalised bovine - cell line. PLoS One 9:e97765
Rehmsmeier M, Steffen P, Hochsmann M, Giegerich R (2004) Fast and effective prediction of microRNA/target duplexes. Rna 10:1507-1517

Riaz A, Dry I, Levy CS, Hopkins J, Grey F, Shaw DJ, Dalziel RG (2014) Ovine herpesvirus-2-encoded microRNAs target virus genes involved in virus latency. J Gen Virol 95:472-480

Russell GC, Stewart JP, Haig DM (2009) Malignant catarrhal fever: a review. Vet J 179:324-335

Schock A, Collins RA, Reid HW (1998) Phenotype, growth regulation and cytokine transcription in ovine Herpesvirus-2 (OHV-2)-infected bovine T-cell lines. Vet Immunol Immunopathol 66:67-81

Sharova LV, Sharov AA, Nedorezov T, Piao Y, Shaik N, Ko MS (2009) Database for mRNA half-life of 19977 genes obtained by DNA microarray analysis of pluripotent and differentiating mouse embryonic stem cells. DNA Res 16:45-58

Umbach JL, Kramer MF, Jurak I, Karnowski HW, Coen DM, Cullen BR (2008) MicroRNAs expressed by herpes simplex virus 1 during latent infection regulate viral mRNAs. Nature 454:780-783

Yang E, van Nimwegen E, Zavolan M, Rajewsky N, Schroeder M, Magnasco M, Darnell JE Jr (2003) Decay rates of human mRNAs: correlation with functional characteristics and sequence attributes. Genome Res 13:1863-1872

Publisher's Note Springer Nature remains neutral with regard to jurisdictional claims in published maps and institutional affiliations. 